\title{
Squaring the circle: The cultural relativity of 'good' shape.
}

Debi Roberson

University of Essex
Jules Davidoff

Goldsmiths College

University of London
Laura Shapiro

Goldsmiths College

University of London

This research is supported by Economic and Social Research Council Grant R0002383-10.

Correspondence concerning this article should be addressed to: Debi Roberson, Department of Psychology, University of Essex, Colchester CO4 3SQ, UK; email: robedd@essex.ac.uk or Jules Davidoff, Department of Psychology, Goldsmiths College, University of London, London SE14 6NW, UK; email: j.davidoff@gold.ac.uk. 


\section{Abstract}

The Gestalt theorists of the early twentieth century proposed a psychological primacy for circles, squares and triangles over other shapes. They described them as 'good' shapes and the Gestalt premise has been widely accepted. Rosch (1973), for example, suggested that shape categories formed around these 'natural' prototypes irrespective of the paucity of shape terms in a language. Rosch found that speakers of a language lacking terms for any geometric shape nevertheless learnt paired-associates to these 'good' shapes more easily than to asymmetric variants. We question these empirical data in the light of the accumulation of recent evidence in other perceptual domains that language affects categorization. A cross-cultural investigation sought to replicate Rosch's findings with the Himba of Northern Namibia who also have no terms in their language for the supposedly basic shapes of circle, square and triangle. A replication of Rosch (1973) found no advantage for these 'good' shapes in the organization of categories. It was concluded that there is no necessary salience for circles, squares and triangles. Indeed, we argue for the opposite because these shapes are rare in nature. The general absence of straight lines and symmetry in the perceptual environment should rather make circles, squares and triangles unusual and, therefore, less likely to be used as prototypes in categorization tasks. We place shape as one of the types of perceptual input (in philosophical terms, 'vague') that is readily susceptible to effects of language variation. 


\section{Introduction}

Whether concepts are linguistically relative or universally independent of natural languages has been debated in psychology, linguistics, anthropology and philosophy for the last 100 years (Boas, 1911/1965; Brown \& Lenneberg, 1954; Whorf, 1956, Vygotsky, 1962; Berlin \& Kay, 1969; Fodor, 1975; Keil, 1992; Carey, 2001). The debate is as lively today as ever it was (Gumperz \& Levinson, 1996; Gopnik, 2001; Spelke \& Tsivkin, 2001; Gentner \& Boroditsky, 2001; Bowerman, 1996, Bowerman \& Choi, 2001; Slobin, 1996).

Early suggestions, such as those of Boas, Sapir and his pupil Whorf, that thought must be linguistically relative arose from well-documented anthropological findings of widespread human linguistic and cultural diversity. It was argued that languages and cultures could not differ as radically as they do if the underlying concepts were universal. Psychological evidence from both within and across cultures also favored tight links between thought and language (Brown \& Lenneberg, 1954; Lenneberg \& Roberts, 1956; Stefflre, Castillo \& Morley, 1966). Nevertheless, an extreme position that the language of thought is nothing except that determined by the speaker's natural language is easy to reject. Clearly, it is possible to translate a concept held in one language into another. So, perhaps not surprisingly, a counter movement began 30 years ago (Berlin \& Kay 1969; Rosch, 1973) that took a diametrically opposite view to the supporters of Whorf. In line with the discoveries being made that the visual system was both inherently modular and its neuronal functions dramatically specific (Hubel \& Wiesel, 1968; Sperry, 1962), it was not difficult to believe that perceptual categories such as color (Berlin \& Kay, 1969; Rosch Heider \& Olivier, 1972) and 
shape (Rosch, 1973) were universal and probably innate. Indeed, Kay \& McDaniel (1978) argued that color categories were based on the properties of the opponentprocess neurones of the visual cortex, They were held to derive from their null points (called unique hues) that acted as prototypes around which categories could form. We now know that prototypical colors do not have a neat relation to unique hues (Saunders \& van Brakel, 1997; Webster et al, 2000).

Berlin and Kay (1969) contended that the linguistic and cultural differences found for perceptual categories arise only because languages fail to fully describe the potential sets of concepts and not because of underlying differences in thought. They suggested that extreme linguistic relativity (i.e., concepts are solely determined by language) could not be true for color because color terms are easily translatable between languages. While this is true for those languages that have approximately the same number of color terms, some ingenuity is required to translate into English the meaning of a term, such as 'nol' in Berinmo (Roberson, Davies \& Davidoff, 2000), that is used to describe half the range of visible colors (about a million justnoticeable-differences) and includes colors that we call green, yellow, blue, brown and purple. Nevertheless, initial experimental research appeared to uphold the hypothesis that cognitive categories of color could exist independently of language. The evidence came from cross-linguistic experiments (Rosch Heider \& Olivier, 1972), infants (Bornstein, Kessen \& Weiskopf, 1976) and macaque monkeys (Sandell, Gross \& Bornstein, 1979). However, a number of methodological problems have been noted with each of these sources of evidence. 
Saunders and van Brakel (1997) provided detailed criticism of the cross-cultural studies. They argued that the cross-linguistic evidence depended heavily on data from speakers of languages with very few color terms who performed extremely poorly on color memory tasks. More recent attempts to replicate and extend Rosch Heider's cross-cultural work have not only failed to find any evidence for a universal set of cognitive color categories but have also found positive evidence of linguistic relativity (Davidoff, Davies \& Roberson, 1999; Roberson et al, 2000). In a paradigm borrowed from Kay \& Kempton (1984) and two additional new paradigms for cross-linguistic studies of color, speakers of Berinmo (a language from Papua New Guinea with only five basic color terms) showed no evidence of a cognitive organization for color categories that did not match their language terms. The Kay and Kempton (1984) paradigm showed that odd-one-out decisions were based, not on innate color categories, but on the Berinmo terms. Additionally, in a recognition memory task, Roberson et al (2000) showed enhanced discrimination of small differences in hue at the boundaries between Berinmo linguistic categories but not at the supposedly innate boundary that distinguishes blue from green. The second of the new paradigms showed, in a task that required categorization of colored stimuli, more efficient learning only when the categories were consonant with their language terms. There was no incipient tendency for divisions between green and blue or between green and yellow to be easily learned. So, adult speakers of different languages appear to have cognitive color categories that exactly match their language categories even when their language contains few color terms.

Adult-like color categories have been reported in pre-linguistic infants and in other primates. The report that human infants have categorical responses to wavelength 
variation (Bornstein et al, 1976) has been criticized on a number of grounds (Banks \& Salapatek, 1984; Werner \& Wooten, 1985). Even if all changes in wavelength were perceptually equal, infants might have been responding to preferences for particular hues, brightnesses or saturations rather than to perceived similarities/dissimilarities of hue. Moreover, these results are at odds with more recent findings that infants are not sensitive to changes in the physical properties (color and/or shape) of a hidden object (Xu \& Carey, 1996). Even at 10 months, infants who see a yellow toy hidden behind a screen, that is subsequently lowered to reveal a red toy, do not look longer at this impossible outcome than they do at the expected outcome - the yellow toy. Xu \& Carey concluded that infants' representations, which they call 'object files', do not carry information about color. The data from infants showing innate color categories must therefore be in some doubt. There is even more doubt concerning the crossspecies evidence for color categories.

Sandell et al (1979) used Munsell colors in their investigation of color categories in monkeys. The conclusion drawn from their study was that monkeys, like humans, have innately driven color categories. They compared generalization responses for cross- and within-category pairs. If monkeys understood that blue was different from green, there ought to be less generalization from a green stimulus to a blue color than to another green; this is what they report. However, in constructing their pairs of stimuli, Sandell et al only considered the dominant wavelength of the Munsell stimuli. Quite different pairs would need to have been chosen to really achieve their aim of equating perceptual differences. As the Munsell notations are given in Sandell et al (1979), it is easy to verify that every one of their cross-category pairs was psychophysically more distant than every comparison within-category pair. 
If color categories are neither universal nor innate, but vary across languages and cultures, despite the apparently tight relation between wavelength and neural mechanisms, then the presumption of universality for other perceptual categories such as that for 'basic' shapes (Rosch, 1973) also bears re-examination. In a pioneering set of experiments Rosch (Rosch Heider \& Olivier, 1972; Rosch Heider, 1972; Rosch, 1973 ) investigated the conceptual structure of natural categories; that is, those "concepts designable by words in 'natural languages'"(Rosch, 1973, p.329). Rosch argued that, rather than all dimensions varying along a continuum, there are clusters of salient attributes in the world (animals tend to have legs and heads, plants tend to have stalks and leaves). She suggested that these "real" (quotation marks in original) categories reflected already-present and non-arbitrary distributions of attributes along dimensions in the physical environment. These 'natural' categories were centered on prototypes within a graded structure that resulted in indistinct ('fuzzy') boundaries. It therefore followed that the prototypes should universally attract attention more readily and individuals should orient towards them in preference to less 'good' examples of a category (Heider, 1971).

In Rosch's view, one such domain was that of shape. Here, the contention was that particularly 'good' forms, in Gestalt terms, (circle, square, triangle), were naturally salient prototypes around which categories of shape would emerge (Rosch, 1973). To test her hypothesis, Rosch (1973) investigated shape concept learning in a remote traditional culture (the Dugum Dani hereafter called 'Dani') of Irian Jaya whose language lacked monolexemic terms for geometric forms (K.G. Heider, 1970). Rosch 
first demonstrated that the Dani showed no pre-experimental bias towards grouping shape stimuli in a particular way. She then taught participants to group variants of these shapes into three categories in a paired-associate learning paradigm. Participants learned one of seven sets of shape variants, for which the circle, square and equilateral triangle were either the central exemplars, or peripheral items in the set. Despite lacking either labels for these shapes or pre-existing perceptual categories, participants learnt the set more easily if the 'good' stimuli were central. Additionally, the 'good' shapes were learnt faster than other stimuli even when they were peripheral to the set. Finally, learners of all sets ranked the 'good' stimuli as better examples of the sets even when they had been peripheral items. In fact, this last pattern of results was only found for circles and squares; for triangles, any three sided, straight-line figure seemed to serve equally well as a best example. Nevertheless, the majority of Rosch's data point to there being non-arbitrary categories of shape that form around perceptually salient natural prototypes even for individuals who encounter such classes of shape stimuli for the first time.

General acceptance of Rosch's thesis that "regular geometric forms, such as circles, squares and triangles are perceptually salient figures" (Bruce \& Green, 1985, p.114) has remained unaffected by observations and theoretical constraints that could easily have led to contrary conclusions. For example, these 'good' forms are extremely rare in the natural world (Hailman, 1977) and infants show no preference for them relative to 'distorted' examples (Bomba \& Siqueland, 1983). Furthermore, while it is not possible to test infants for their ability to sort shapes, the evidence from older children does not encourage the view that circles, squares and triangles are particularly salient. 
Inhelder and Piaget (1964) reported a series of experiments in which younger children (under 4 yrs) failed to group circles, squares and triangles, but rather made continuous patterns with the stimuli, appearing to shift their matching criterion from shape to pattern to size between each pair of examples chosen. When asked to draw figures such as circles and triangles the children only represented the topological relations and would often ignore the Euclidean relations. For example, they were quite prepared to draw a circle when copying a triangle. Older children (4-5 years), when asked to group the stimuli tended to make patterns representative of 'collective objects', for example by placing two circles under a rectangle for a car. Piaget suggested that younger children could not sustain the concept of overall similarity across space and time to form a group and lacked the ability to differentiate "the logical structures characteristics of discontinuous sets from the sub-logical structures characterizing the subdivision of a continuous whole" (Inhelder \& Piaget, 1964, pp 46).

More recently, Abecassis et al. (2001) found that, although English-speaking adults generalize a novel object name only to new exemplars from the same shape category, children under 5 years of age are just as likely to generalize the name to objects from a different shape category. Such apparent insensitivity to 'basic' shape categories is in marked contrast to the competence exhibited by children of the same age in matching objects of the same spatial ratio (length: width) even in the face of similarly configured alternatives. Gibson (1969) suggested that Gestalt principles of perceptual grouping by similarity develop with age and experience. If such groupings are late 
acquired, and not present pre-linguistically, it seems unlikely that they would be universally present, especially in individuals whose language has no terms for them. It is not only the developmental data that casts doubt on the saliency of circles, triangles and squares; there are also theoretical concerns. Human observers interpret 2-D shapes as representations of three-dimensional (3-D) objects in the world (Sinha \& Poggio, 1996, Pylyshyn, 1999). It is just those 2-D figures that are most asymmetrical, complex and discontinuous that are most likely to be perceived as the projection of 3-D figures (Hochberg \& Brooks, 1960). Thus, given the tendency of objects in the natural world to have irregular shapes, symmetrical geometric forms ought to lack rather than demand perceptual saliency. Willats (1992) also re-examined the basis on which shape categories might be considered 'natural' and suggested that there is only one overriding shape property that might be universal - that of extendedness. Following Marr and Nishihara's generalized cone theory (1978) and Biederman's (1987) geon theory, Willats sought a set of perceptual primitives that would allow 3-D interpretation of the silhouettes of irregular natural objects such as trees and clouds. Willats suggested that 2-D images are interpreted as 3-D shapes by making an implicit assumption about their relative extensions in 3-D space. 2-D representations (whether regular or irregular) would be interpreted as either 'lumps' or 'sticks' depending on the likelihood of their 3-D extension. 'Lumps' (in which circles, squares and triangles would be included as well as irregular shapes like stones or fruits) are likely to be saliently extended in both directions, whereas 'sticks' (long objects) are more likely to be extended in only one direction. Distinguishing between 'long' and 'round' objects in the world can thus be carried out very simply and with great accuracy on the basis of the retinal image in all but the most exceptionally unusual views of a static image (e.g. an end-on view of a stick). If the 'lump' vs 
'stick' distinction is the only universal discriminant needed to categorize objects in the natural world, languages lacking three separate labels for circle, square and triangle should not show enhanced learning for 'good' forms over other variants. So, with both theoretical and empirical data that do not conform to Rosch's views concerning universal cognitive categories, we re-examined the independence of shape categorization from language.

The present study attempted to replicate Rosch's (1973) findings. The pastoral Himba people of northern Namibia, whose language lacks terms for any of these geometric shapes were given the full range of tasks set out in Rosch (1973) in the course of a larger cross-cultural study.

\section{Replication of Rosch (1973)}

\section{Examination of pre-experimental shape categories}

These studies investigated whether the Himba, despite lacking linguistic categories, showed any tendency to group stimuli into circles, squares and triangles. In the original Rosch (1973) study, 7 Dani participants were asked to free-sort a random arrangement of the 21 figures in figure 1A into as many groups as they felt appropriate. Rosch also asked 9 participants to carry out an oddity task using three of the stimuli. Two of the shapes were variants of one 'good' form and the third shape was a variant of another. The participants had to select the two shapes that were most similar. In both tasks, grouping of variants of the same form was at chance. 
In the present study, 7 Himba adults conducted Rosch's free-sorting task. The number of groups formed (for the 21 stimuli) varied from 3 to 11 . The only participant who spontaneously arranged the stimuli into three groups produced a group that contained all three types of shape. Grouping of variants of the same form was not different from chance $(z=.74, p>.1)$. Another 7 Himba adults carried out Rosch's oddity task. Each participant saw all 21 shapes in this way. Pairing of two variants of the same shape (e.g., two triangles) was significantly above chance $(z=2.10, p<.05)$. Our result differed from that reported by Rosch, but is consistent with judgments based on perceptual similarity.

Rosch also investigated whether the Dani could use verbal circumlocutions to describe the forms. In a two-person communication task, an identical set of 21 forms was laid out in front of each person in different random orders with a screen placed between them. One person was designated as the speaker and had to describe each shape so that the listener could choose it from their set. Listener's errors were randomly distributed amongst the three form types. In the present study, 4 pairs of Himba adults were tested. Like the Dani, Himba participants described the shapes in terms of specific objects (e.g., "kraal", "milk container"). Of 52 descriptions, only 18 responses were from the correct shape category. It thus seemed safe to conclude that the Himba, like the Dani, were not predisposed to group the sets of 2-D figures according to 'good' shapes, and to proceed with the main learning study.

Paired-associate learning. 
Twenty-eight monolingual Himba adults completed learning to criterion ( 8 males, 20 females ranging in age between approx. 15 and $40 \mathrm{yrs}$ ). None had participated in the pilot studies.

\section{Stimuli}

The same seven sets of shapes were used as in Rosch (1973). The first Set (illustrated in figure 1A) consisted of (a) 'good' stimuli (circle, square and triangle) with 6 transformations on the 'good' form. The prototypes for the other 6 Sets were obtained from the transformations used in Set 1. For all Sets there were three figures acting as the new prototypes and the transformations were carried out on these new prototypes. So, in Set 2 (see Fig 1B), the gapped figures from set 1 were the prototypes and all the other above transformations were carried out on gapped figures. Set 3 used the lineto-curve transformations from set 1 as prototypes and so on for the remaining Sets. The 'good' stimuli ('natural' prototypes) were also included in every set in place of the prototype for that set. So, for example, in Set 2, the 'good' stimulus was created by closing the gap on the prototype. Figures were approx. $2 \mathrm{cms}$ in area and were mounted on $5 \mathrm{~cm} \times 5 \mathrm{~cm}$ white card. Paired-associate relationship terms used were "Dati" (father), "Mama" (mother) and "Omuage" (child).

\section{Procedure}

Each learning set contained 21 stimuli (7 'circles', 7 'squares' and 7 'triangles'). Each participant learnt only one set (for example those in Fig 1A or those in Fig 1B). 
Participants learnt a paired-associate name as the correct response to each of the 7 stimuli in a category. In the first instance, all 21 stimuli were laid out in front of the participant in three groups ('circles', 'squares' and 'triangles'). The experimenter pointed to each shape in turn and named it with a Himba kinship term. Name to shape matching was counterbalanced across participants. The same 21 stimuli were then shown in a different random order on each test; with five tests a day and feedback after each response. Stimuli were presented in random orientation. The criterion for success was the correct naming of all 21 stimuli in the set.

( Figs. 1A \& 1B about here)

When criterion was reached, a transfer task was given in which the participant was required to name three new stimuli, a 'circle', 'square' and 'triangle' from a different set. Subsequently, for 11 of the participants, estimates were obtained of the best examples for each category. All the stimuli were laid in front of the participant and the experimenter asked: "Which is the best (Omuage)? Which one looks exactly like (Omuage)?".

Results

Four Himba participants completed sets 1, 2, 3, 4 and 6, five completed set 5 and three completed Set 7. The Himba made fewer errors to criterion than the Dani on all sets. However, on the transfer task, only 17/28 named all three transfer shapes correctly and Himba participants made $18 \%$ errors compared to $2 \%$ made by the 
Dani. Table 1 shows, compared to Dani data, (i) the mean errors to criterion for each set of stimuli (defined by prototype) (ii) the mean errors to criterion per Transformation type and (iii) mean rank of best example per Transformation type.

Error scores for each stimulus were analyzed in a 7(Set type (type of prototype): 1-7) x 7(Transformation type: a-g (see Fig. 1)) x 3(Shape type: circle v square v triangle) mixed design ANOVA. It revealed no significant effect of Set type: $F(6,21)=1.83$, $\mathrm{MSE}=12.86, \mathrm{p}>.1$, a significant effect of Transformation type: $\mathrm{F}(6,126)=8.84$, $\mathrm{MSE}=3.22, \mathrm{p}<.001$ and a significant effect of Shape type $\mathrm{F}(2,42)=12.36, \mathrm{MSE}=$ $1.04, \mathrm{p}<.001$. In addition, there was a significant interaction between Set type and Shape type: $\mathrm{F}(12,252)=4.56, \mathrm{MSE}=1.619, \mathrm{p}<.001$, a significant interaction between Transformation type and Shape type: $\mathrm{F}(12,252)=3.68, \mathrm{MSE}=1.62, \mathrm{p}<$ .001 , as well as a significant three-way interaction: $\mathrm{F}(72,252)=1.85, \mathrm{MSE}=1.62, \mathrm{p}<$ .001 .

To explore the three-way interaction, separate 7(Set type: 1-7) x 7(Transformation type: a-g) mixed design ANOVAs were carried out for each Shape type. The analysis for circles revealed a significant effect of Set type: $F(6,21)=3.41, \operatorname{MSE}=3.77, \mathrm{p}<$ .05 , a significant effect of Transformation type: $F(6,126)=10.05, \mathrm{MSE}=1.22, \mathrm{p}<$ .001 and a significant interaction: $\mathrm{F}(36,126)=2.36, \mathrm{MSE}=1.22, \mathrm{p}<.001$. NewmanKeuls pairwise comparisons showed differences for comparisons involving 'good' stimuli and also for comparisons that did not concern the 'good' stimuli. In the former case, the comparisons did not always favor the 'good' stimuli. 
Considering within-set differences: in Set 1, pairings to the gapped stimulus were learnt more slowly than to the two-line, one-line extended, irregular, and curved shapes ( $\mathrm{p}<.01, \mathrm{p}<.01, \mathrm{p}<.01, \mathrm{p}<.05$ respectively) and also to the 'good' stimulus $(\mathrm{p}<.05)$. In Set 2 , pairings to the freehand shape were learnt significantly more slowly than to the irregular, curved and one-line extended shapes (all ps $<.05$ ). In Set 5 (two lines extended prototype) and Set 6 (irregular figure prototype), pairings to the 'good' stimulus were learnt more slowly than to all other shapes (all ps $<.01$ ).

Considering between-set differences: the only reliable effects were that pairings to the 'good' stimulus were learnt significantly slower $(\mathrm{p}<.05)$ in Set 6 (irregular figure prototype) than in Set 7 (where the freehand shape was prototype).

A similar analysis for squares revealed no significant effect of Set type: $F(6,21)=$ $1.66, \mathrm{MSE}=11.05, \mathrm{p}>.1$, but a significant effect of Transformation type: $\mathrm{F}(6,126)=$ $3.48, \mathrm{MSE}=2.31, \mathrm{p}<.01$ and no significant interaction $\mathrm{F}(36,126)=1.16, \mathrm{MSE}=$ $2.31, \mathrm{p}>.2$. Newman-Keuls pairwise comparisons revealed that pairings to the 'good' stimulus were learnt significantly more slowly than to either the curved or two-line extended shapes (both ps $<.01$ )

The analysis for triangles revealed a similar but more pronounced pattern. There was no significant effect of Set type: $\mathrm{F}(6,21)=1.96, \mathrm{MSE}=8.94, \mathrm{p}>.1$, but a significant effect of Transformation type: $\mathrm{F}(6,126)=2.17, \mathrm{MSE}=1.46, \mathrm{p}<.05$ and no significant interaction $\mathrm{F}(36,126)=1.26, \mathrm{MSE}=1.46, \mathrm{p}>.1$. Post-hoc tests showed that pairings to the 'good' stimulus were learnt more slowly than to all other transformations (all ps $<.01$ ). 
Rosch carried out separate analyses on each of Set type, Transformation type and Shape type. So, it is impossible to compare the similarity of the interactions in the present data with those obtained with the Dani.

(Table 1 about here)

In Rosch (1973), the Dani were significantly more likely to choose the 'good' stimuli as best example of the category, regardless of whether they had been the central or peripheral examples in the set. In the present study, the mean ranks assigned to the seven shape transformations were analyzed in a repeated measures ANOVA. It revealed a significant main effect of Transformation type: $\mathrm{F}(6,60)=3.55$, MSE $=$ $126.20, \mathrm{p}<.01$. The 'good' stimulus was the transformation most often chosen as the best example. Of these choices, $6 / 11$ chose the square, $4 / 11$ the circle and 7/11 the

equilateral triangle as best example of the category. However, none of the participants who saw Set 1, for which the 'good' stimuli were the prototypes, ranked them as best example. Newman-Keuls pairwise comparisons revealed that the 'good' stimuli was ranked as better examples of the categories than the freehand $(\mathrm{p}<.01)$, irregular $(\mathrm{p}<$ $.05)$, and curved $(\mathrm{p}<.05)$ variants, but there were no other significant differences. Finally, while Dani ratings of best example were related to performance in the pairedassociate learning task, in the present study, ratings of best example did not correlate well with the number of learning errors for each stimulus (all ps $>.08$ ).

\section{Discussion}

The present study attempted to replicate Rosch (1973) in which evidence was found for 'natural' shape prototypes. The experiment required many days for participants to reach criterion and was difficult to administer to the Himba who have duties that take 
them away from their villages. Thus, it was not possible to test the numbers that Rosch managed with the Dani. However, a sufficient number were tested to be sure that there is no trend towards a superiority for 'natural shape prototypes'. The Himba, who have no words for the regular geometric shapes (circle, square, triangle), learnt categories based around irregular shapes with no more difficulty than categories based around 'good' shapes. In fact, rather the reverse. The effects of type of transformation differed across the three shape categories but it was very seldom the case that pairings to the 'good' stimulus were learnt faster than to other shapes in the set. Indeed, for the 'good' triangle, in particular, pairings were learnt more slowly. Rosch's conclusion that "there are nonarbitrary ... form categories which form around perceptually salient 'natural prototypes'" (1973, p348) seems unwarranted in the light of that evidence.

There might have been reasons associated with the different testing situations that might explain the differences between our results and those of Rosch with the Dani. It certainly could not be that the Himba were less motivated because they reached criterion in fewer trials. However, it could be that the learning mechanisms differed. The Himba seemed more inclined to judge similarity on perceptual grounds. In the examination of pre-experimental categories, the Himba made judgments of odd-oneout based on perceptual similarity. Rather surprisingly, the Dani did not, although Rosch (personal communication) reports that Dani participants seemed particularly interested in geometrical and symmetrical objects and that they spent much time around the experimenter's house examining the test stimuli (and other symmetrical objects such as buttons and notebooks); exclaiming and running their fingers over them. In contrast, the Himba only saw the test stimuli during the course of the experiment and thus came to the test situation unfamiliar with the composition of the 
sets. The transfer task also suggests that the Himba had not learned the categorization task by forming a prototype. After having learned the categories, the Dani reliably transferred names to new stimuli on a categorical basis. Almost half of the Himba participants made at least one categorical error in the transfer task. If they were attempting to generalize names on the basis of perceptual similarity, a few mistakes were inevitable.

Perceptual similarity can be an effective mechanism for categorization as shown by adults who have lost language. Many categories of objects (e.g., trees compared to cars) are so different that the aphasic patient can solve the categorization task purely on perceptual similarity (Goldstein, 1948; Roberson, Davidoff \& Braisby, 1999). The same is true for the pre-linguistic child. For example, a one-year-old child is quite capable of producing object files (Treisman, 1988; Rakison \& Butterworth, 1998a,b; Carey, 2001) without the need for linguistic input. Indeed, even the linguistic child may still be governed by perceptual similarity in category decisions. Three-year-old children do not generalize names for shapes in a proper categorical (and hence linguistic) basis (Abecassis et al., 2001). The categorical tasks in Abecassis et al were similar to those used in Roberson et al (2000); they contrasted perceptual with categorical similarity. Abecassis et al found that, although English-speaking adults generalize a novel object name only to new exemplars from the same shape category, children are just as likely to generalize the name to objects from a different shape category if the objects are equally perceptually similar. Abecassis et al further found that adults do not always treat shape differences categorically. When learning names for novel shapes, adults treat degrees of curvature and parallelism as categorical but, when judging the similarity of objects, as belonging to continua of change. This 
contrast of behavior suggests that it is the act of linguistic categorization that causes shapes to be perceived categorically. It would appear that our Himba participants had a tendency to behave more like children who have yet to acquire linguistic categories or like adults performing a perceptual similarity task. Thus, we conclude, that the Himba are driven in their shape categorization solely by perceptual similarity.

The present data therefore favor cultural and linguistic relativity for shape categorization and find no inevitable preference for categories to be based around the 'good' forms of Gestalt perceptual analysis. Another Gestalt principle -that of similarity- might also not be universal. Gibson (1969) reviewed experiments in which five-to-eight year-olds were asked to sort different basic shapes into groups. Younger children, and those with developmental delays, simply put the stimuli in rows without sorting them. Gibson (1969) suggested that Gestalt principles of perceptual grouping by similarity develop with age and experience. If such groupings are late acquired, it seems unlikely that they would be universally present especially in individuals whose language has no terms for them. However, it is important to stress, in order to avoid misunderstanding, that the present results do not imply that other Gestalt principles of perceptual grouping (e.g., proximity, closure and common fate) are not universal. These Gestalt principles refer to processes quite different to shape categorization (Han \& Humphreys, 1999, Pylyshyn, 1999). Indeed, evidence from both normal and braindamaged populations show that perceptual grouping is a process independent of shape identification or discrimination (Trick \& Enns, 1997; Kartsounis \& Warrington, 1991; Davidoff \& Warrington, 1993). For example, reasonably preserved shape discrimination can co-exist with greatly impoverished grouping by Gestalt organizational principles (Kartsounis \& Warrington, 1991). Thus, it is not Gestalt 
principles of perceptual grouping that determine shape categorization and, in consequence, they are not germane to the present studies. Our data only question the Gestalt notion of universal 'good' shapes.

The one aspect of our results that somewhat conforms to the pattern found in Rosch (1973) is that the Himba rated the 'good' stimuli as best examples of the categories. However, the preference for the 'good' stimuli was less pronounced than for the Dani and had no behavioral consequences for learning. Nevertheless, the Himba 'best example' preferences appear peculiar. It seems odd that any of the Himba rated the stimuli they found most difficult to learn as the best examples. It is, therefore, possible that there was a problem in the translation. As the Dani language contained no term for 'best example' this was translated to the Dani using a verb form used for 'usual or typical' activities. There is no equivalent verb in Himba but this was thought immaterial, as it was possible to use a direct translation of 'best example'. The Himba term ought to have been more coherent but it may have been somewhat misunderstood. We can only infer that some of the Himba interpreted the term 'best example' to reflect the status of the 'good' stimulus as most unusual.

Though there is clearly a need for further cross-cultural investigations of shape categorization, there is no disputing that this study has failed to replicate the main findings of Rosch (1973). We argue that the failure to replicate is not unexpected given the type of stimuli that are employed in the shape categorization task. In fact, consideration of stimulus type and task may enhance our general understanding of when language is involved in categorization. For example, it is not disputed that objects (e.g., natural kinds) have many properties that would ensure equivalent 
classification in all cultures (Atran, 1990; Berlin, 1992). We would make the case that language becomes involved when stimuli are, in philosophical terms, vague

(Dummett, 1975); this is necessarily true for color and the argument is illustrated with color to show that perceptual similarity is an insufficient procedure for the production of categories.

Take the case of a series of color patches of decreasing wavelength, each of which is indistinguishable from its immediate neighbors because the steps in wavelength are below discrimination threshold for the human visual system. How is that series of color patches to be divided into different categories? Let us say that it is agreed that one end patch can be called 'red'. But, then so must its (indistinguishable) immediate neighbor. Pursuing the reasoning for adjacent stimuli, one arrives at the paradoxical conclusion that all colors in the series (even 'blues' at the other end) must be called 'red'. Thus, so as not to be trapped in the paradox, it is held that a non-perceptual mechanism must be required to form categories from such continuously varying perceptual stimuli (Dummett, 1975; Wright, 1975). We would argue that the mechanism is language.

It is not only color for which there is vagueness with respect to similarity. There are several other important classes of perceptual stimuli for which categorization is likewise susceptible to the effects of language and language differences. These include number (Brysbaert, Fias \& Noel, 1998), space (Wassman \& Dasen, 1998; Levinson, 1996, Bowerman, 1996) and time (Weist et al., 1997). Cognitive categories for shapes, if truly universal and independent of linguistic categories, would be a surprising exception. Consider the difficulty of judging whether two stones are from 
the same shape category. Judging the shape category for a stone that has three continuously varying independent dimensions (height, width and depth) is difficult by observation alone. In fact, as is seen with color, impossible. There would be a clear evolutionary advantage for a species that could communicate the similarity for shapes of stones with some words or symbols. Indeed, one might even be prepared to argue that language evolved in our species in order to solve the many such communication problems that cannot be solved by perceptual similarity alone.

In summary, circles, triangles and squares have a special place in mathematics but such regularity and symmetry of shape is rare in the natural world (Hailman, 1977). Our findings indicate that Western categories of 'circle' 'square' and 'triangle' arise through cultural and linguistic agreement. The concepts 'square', 'circle' and 'triangle' appear to be learned only effortfully and with the assistance of language. The Himba culture has not yet found it necessary to distinguish these shape categories linguistically from one another. From the verbal descriptions offered in the circumlocution task (e.g., "building for drying maize", "water can", "side of a mountain"), it seems probable that all the variants of the three forms were interpreted as representations of 3-D objects. This interpretation would put all the shape variants tested into the general category of "lumps" (Willats, 1992) and thus all variants might be equally good exemplars of a type of "lump". Rather than reflecting natural discontinuities in the world, it would appear that "we cut nature up (and) organize it into concepts ... largely because ... we are parties to an agreement to do so, not because nature itself is segmented in exactly that way for all to see" (Whorf, 1956, pp241). 
References:

Abecassis, M., Sera, M.D., Yonas, A. \& Schwade, J. (2001) What's in a shape? Children represent shape variability differently than adults when naming objects. Journal of Experimental Child Psychology, 78, 213-239.

Atran, S. (1990) Cognitive foundations of natural history. New York: Cambridge University Press.

Banks, M.S. \& Salapatek, P. (1984) Infant visual perception. In: P.H. Mussen (Ed.), Handbook of Child Psychology, Vol II. (4 ${ }^{\text {th }}$ Edition pp 525-532) New York: John Wiley \& Sons.

Berlin, B. (1992) Ethnobiological classification: Principles of categorization of plants and animals in traditional societies. Princeton, NJ: Princeton University Press.

Berlin, B. \& Kay, P. (1969) Basic color terms: Their universality and evolution. Berkeley: University of California Press.

Biederman, I. (1987) "Recognition-by-components: A theory of human image understanding". Psychological Review, 94, 115-147.

Boas, F. (1965[1911]) The mind of primitive man. Reprint: New York: The Free Press.

Bomba, P.C. \& Siqueland, E.R. (1983). The nature and structure of infant form categories. Journal of Experimental Child Psychology, 35, 609-636.

Bornstein, M.H., Kessen, W. \& Weiskopf, S. (1976) Color vision and hue. categorization in young human infants. Journal of Experimental Psychology: Human Perception and Performance, 2, 115-129. 
Bowerman, M (1996) The origins of children's spatial semantic categories: Cognitive versus linguistic determinants. In: J.J. Gumperz \& S.C. Levinson (Eds.) Rethinking linguistic relativity. Cambridge: Cambridge University Press.

Bowerman, M. \& Choi, S. (2001) Shaping meanings for language: universal and Language specific in the acquisition of spatial semantic categories. In: M.

Bowerman \& S. Levinson (Eds.), Language acquisition and conceptual development. Cambridge: Cambridge University Press.

Brown, R. \& Lenneberg, E. A study in language and cognition. Journal of Abnormal and Social Psychology, 49, 454-462.

Brysbaert, M., Fias, W. \& Noel, M-P. (1998) The Whorfian hypothesis and numerical cognition: is 'twenty-four' processed in the same way as 'four-and-twenty'? Cognition, 66, 51-77.

Bruce, V. \& Green, P.R. (1985) Visual perception: Physiology, psychology and ecology. London: Lawrence Erlbaum Associates.

Carey, S. (2001) Whorf versus continuity theorists: Bringing data to bear on the debate. In: M. Bowerman \& S. Levinson (Eds.), Language acquisition and conceptual development. Cambridge: Cambridge University Press.

Davidoff, J., Davies, I. \& Roberson, D. (1999) Color categories of a stone-age tribe. Nature, 398, 203-204.

Davidoff, J. \& Warrington, E. K (1993). A dissociation of shape discrimination and figure-ground perception in a patient with normal acuity. Neuropsychologia, 31, 8393.

Dummett, M. (1975) Wang's Paradox. Synthese, 30, 301-324.

Fodor, J.A. (1975) The language of thought. Cambridge, MA: Harvard University Press. 
Gentner, D. \& Boroditsky, L. (2001) Individuation, relativity and early word learning. In: M. Bowerman \& S. Levinson (Eds.), Language acquisition and conceptual development. Cambridge: Cambridge University Press.

Gibson, E.J. (1969) Principles of Perceptual Learning and Development. Englewood Cliffs, NJ: Prentice-Hall, Inc.

Goldstein, K (1948). Language and language disturbances. New York: Grune \& Stratton.

Gopnik, A. (2001) Theories, language and culture: Whorf without wincing. In: M. Bowerman \& S. Levinson (Eds.), Language acquisition and conceptual development. Cambridge: Cambridge University Press.

Gumperz, J.J. \& Levinson, S.C. (1996) Rethinking linguistic relativity. Current Anthropology, 32, 613-623.

Hailman, J.P. (1977) Optical signals: Animal communication and light. Bloomington: Indiana University Press.

Han, S.H. \& Humphreys, G.W. (1999) Interactions between perceptual organization based on Gestalt laws and those based on hierarchical processing. Perception \& Psychophysics, 61, 1287-1298.

Heider, K.G. (1970) The Dugum Dani. New York: Aldine.

Heider, E. (1971) "Focal" color areas and the development of color names. Developmental Psychology 4, 477-455.

Hochberg, J. \& Brooks, V. (1960) The psychophysics of form: Reversible perspective drawings of spatial objects. American Journal of Psychology, 73, 337-354.

Hubel, D.H. \& Weisel, T.N. (1968). Receptive fields and functional architecture of monkey striate cortex. Journal of Physiology, 195, 215-243. 
Inhelder, B. \& Piaget, J. (1964) The early growth of logic in the child. London: Routledge \&Kegan Paul Ltd.

Kartsounis, L.D \& Warrington, E. K.(1991) Failure of object recognition due to a breakdown of figure-ground discrimination in a patient with normal acuity. Neuropsychologia, 29, 969-980.

Kay, P. \& McDaniel, C.K. (1978) The linguistic significance of the meanings of basic colour terms. Language, 54, 610-646.

Kay, P. \& Kempton, W. (1984) What is the Sapir-Whorf hypothesis? American Anthropologist, 86, 65-78.

Keil, F.C. (1992) Concepts, kinds and cognitive development. Cambridge, Mass: MIT Press.

Lenneberg, E.H. \& Roberts, J. M. (1956). The language of experience: a study in methodology. Indiana University Publications in Anthropology and Linguistics Memoir 13: supplement to International Journal of American Linguistics 22, 2. Baltimore: Waverly Press.

Levinson, S.C. (1996) Language and space. Annual Review of Anthropology, 25, 353382.

Marr, D. \& Nishihara, H.K. (1978) Representation and recognition of the spatial organization of three-dimensional shapes. Proceedings of the Royal Society of London, Series B, 200, 269-294.

Pylyshyn, Z (1999) Is vision continuous with cognition? The case for cognitive impenetrability of visual perception. Behavioral \& Brain Sciences, 22, 341-423.

Rakison, D.H. \& Butterworth, G. E (1998a). Infants' use of object parts in early categorization. Developmental Psychology, 34, 49-62.

Rakison, D.H. \& Butterworth, G. E (1998b). Infants' attention to object structure in 
early categorization. Developmental Psychology, 34, 1310-1325.

Roberson, D., Davies I. \& Davidoff, J. (2000) Color categories are not universal: Replications and new evidence from a Stone-age culture. Journal of Experimental Psychology: General, 129, 369-398.

Roberson, D., Davidoff, J. \& Braisby, N. (1999) Similarity and categorization: Neuropsychological evidence for a dissociation in explicit categorization tasks. Cognition 71, 1-42.

Rosch, E.H. (1973) Natural categories. Cognitive Psychology, 4, 328-350.

Rosch Heider, E.. (1972) Universals in color naming and memory. Journal of Experimental Psychology, 93, 10-20.

Rosch Heider, E.. \& Olivier, D.C. (1972) The structure of the color space in naming and memory for two languages. Cognitive Psychology, 3, 337-354.

Sandell, J.H., Gross, C.G. \& Bornstein, M.H. (1979) Color categories in Macaques. Journal of Comparative and Physiological Psychology, 93, 626-635.

Saunders, B.A.C. \& van Brakel, J. (1997) Are there non-trivial constraints on color categorization? Behavioral \& Brain Sciences, 20, 167-228.

Sinha, P. \& Poggio, T. (1996) Role of learning in three-dimensional form perception. Nature, 384, 460-463.

Slobin, D.I. (1996) From "thought and language" to "thinking for speaking”. In J.J. Gumperz \& S.C. Levinson (Eds.), Rethinking linguistic relativity. Cambridge,MA: Cambridge University Press.

Spelke, E.S., \& Tsvikin, S. (2001) Initial knowledge and conceptual change: space and number. In: M. Bowerman \& S. Levinson (Eds.), Language acquisition and conceptual development. Cambridge: Cambridge University Press. 
Sperry, R.W. (1962). Some general aspects of hemispheric integration. In

V.B.Mountcastle (Ed.) Interhemispheric relations and cerebral dominance. Baltimore: Johns Hopkins Press.

Stefflre, V., CastilloVales, V. \& Morley, L. (1966). Language and cognition in the Yucatan: A cross cultural replication. Journal of Personality and Social Psychology, $4,112-115$.

Treisman. A.M. (1988). Features and objects. Quarterly Journal of Experimental Psychology, 40A, 201-237.

Trick, L.M. \& Enns, J.T. (1997) Clusters precede shapes in perceptual organization. Psychological Science, 8, 124-129.

Vygotsky, L.S. (1962). Thought and speech. Cambridge, MA: MIT Press.

Wassmann, J. \& Dasen, P.R. (1998) Balinese spatial orientation: Some empirical evidence of moderate linguistic relativity. Journal of the Royal Anthropological Institute, 4, 689-711.

Webster, M.A., Miyahara, E., Malkoc, G. \& Raker, V.E. (2000) Variations in normal color vision. II. Unique hues. Journal of the Optical Society of America, 17, 15451555.

Weist, R.M., Lytinen, P., Wysocka, J. \& Atanassova, M. (1997) The interaction of language and thought in children's language acquisition: A crosslinguistic study. Journal of Child Language, 24, 81-121.

Werner, J.S. \& Wooten, B.R. (1985) Unsettled issues in infant color vision. Infant Behavior and Development, 8, 99-107.

Whorf, B.L. (1956) The relation of habitual thought and behavior to language. In: 
Language, Thought and Reality: Essays by B.L. Whorf. J.B. Carroll (ed.)

Cambridge: M.I.T. Press.

Willats, J. (1992) Seeing lumps, sticks and slabs in silhouettes. Perception, 21, 481496.

Wright, C. (1975) On the coherence of vague predicates. Synthese, 30, 325-365.

Xu, F. \& Carey, S. (1996) Infants' metaphysics: the case of numerical identity.

Cognitive Psychology, 30, 111-153.

Table 1. (i) Mean errors to criterion for each Set of stimuli compared to those made by the Dani (shown in brackets underneath) (Rosch, 1973); (ii) mean errors to criterion per stimulus type and (iii) mean rank of best example per stimulus type.

(i)

Set type (defined by type of prototype)

\begin{tabular}{|c|c|c|c|c|c|c|}
\hline Basic & Gap & $\begin{array}{l}\text { Line-to- } \\
\text { curve }\end{array}$ & $\begin{array}{l}\text { 1-line } \\
\text { extended }\end{array}$ & $\begin{array}{l}\text { 2-line } \\
\text { extended }\end{array}$ & Irregular & Freehand \\
\hline
\end{tabular}

Mean errors per stimulus per person for each Set

\begin{tabular}{cccccccc}
\hline \multicolumn{7}{l}{ Transformation types combined within Sets. Collapsed over circle, square and triangle. } \\
\hline Himba & $\mathbf{2 . 0 2}$ & $\mathbf{1 . 8 8}$ & $\mathbf{0 . 4 2}$ & $\mathbf{1 . 5 1}$ & $\mathbf{1 . 3 3}$ & $\mathbf{1 . 7 6}$ & $\mathbf{1 . 6 5}$ \\
$($ Dani) & $(2.62)$ & $(4.07)$ & $(4.32)$ & $(3.91)$ & $(3.78)$ & $(5.88)$ & $(7.47)$ \\
\hline
\end{tabular}

(ii)

Transformation type

$\begin{array}{llllll} & & \text { Line-to- } & \text { 1-line } & \text { 2-line } \\ \text { Basic } & \text { curve } & \text { extended } & \text { extended } & \text { Irregular } & \text { Freehand }\end{array}$

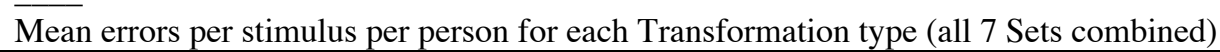

\begin{tabular}{|c|c|c|c|c|c|c|c|}
\hline \multicolumn{8}{|c|}{ Collapsed over the three shape categories } \\
\hline $\begin{array}{r}\text { Himba } \\
\text { (Dani) }\end{array}$ & $\begin{array}{c}2.31 \\
(1.97)\end{array}$ & $\begin{array}{c}\mathbf{1 . 6 7} \\
(3.87)\end{array}$ & $\begin{array}{c}\mathbf{1 . 0 7} \\
(3.86)\end{array}$ & $\begin{array}{c}\mathbf{1 . 3 1} \\
(3.11)\end{array}$ & $\begin{array}{c}\mathbf{1 . 1 6} \\
(2.92)\end{array}$ & $\begin{array}{c}\mathbf{1 . 4 3} \\
(6.07)\end{array}$ & $\begin{array}{c}\mathbf{1 . 5 6} \\
(7.29)\end{array}$ \\
\hline \multicolumn{8}{|c|}{ By shape category } \\
\hline $\begin{array}{l}\text { Circle } \\
\text { HIMBA } \\
\text { (Dani) }\end{array}$ & $\begin{array}{c}\mathbf{1 . 5 7} \\
(0.57)\end{array}$ & $\begin{array}{c}\mathbf{1 . 3 2} \\
(1.71)\end{array}$ & $\begin{array}{c}\mathbf{1 . 0 4} \\
(1.40)\end{array}$ & $\begin{array}{c}\mathbf{1 . 0 7} \\
(1.79)\end{array}$ & $\begin{array}{c}\mathbf{1 . 4 3} \\
(1.67)\end{array}$ & $\begin{array}{c}\mathbf{1 . 8 9} \\
(6.02)\end{array}$ & $\begin{array}{c}\mathbf{1 . 1 4} \\
(6.24)\end{array}$ \\
\hline $\begin{array}{c}\text { Square } \\
\text { HIMBA } \\
\text { (Dani) }\end{array}$ & $\begin{array}{c}\mathbf{2 . 9 3} \\
(2.06)\end{array}$ & $\begin{array}{c}2.32 \\
(6.29)\end{array}$ & $\begin{array}{c}\mathbf{1 . 5 0} \\
(4.30)\end{array}$ & $\begin{array}{c}\mathbf{2 . 3 2} \\
(4.47)\end{array}$ & $\begin{array}{c}\mathbf{1 . 5 0} \\
(4.24)\end{array}$ & $\begin{array}{c}\mathbf{1 . 9 3} \\
(8.57)\end{array}$ & $\begin{array}{c}\mathbf{2 . 2 5} \\
(9.21)\end{array}$ \\
\hline $\begin{array}{c}\text { Triangle } \\
\text { HIMBA } \\
\text { (Dani) }\end{array}$ & $\begin{array}{c}\mathbf{2 . 4 3} \\
(3.27)\end{array}$ & $\begin{array}{c}\mathbf{1 . 3 6} \\
(3.58)\end{array}$ & $\begin{array}{c}\mathbf{0 . 6 8} \\
(5.86)\end{array}$ & $\begin{array}{c}\mathbf{0 . 5 4} \\
(3.09)\end{array}$ & $\begin{array}{c}\mathbf{0 . 5 4} \\
(2.84)\end{array}$ & $\begin{array}{c}\mathbf{0 . 4 6} \\
(3.62)\end{array}$ & $\begin{array}{c}\mathbf{1 . 2 9} \\
(6.43)\end{array}$ \\
\hline
\end{tabular}


(iii)

Transformation type

Basic Gap $\quad \begin{gathered}\text { Line-to- } \\ \text { curve }\end{gathered} \quad \begin{gathered}\text { 1-line } \\ \text { extended }\end{gathered} \begin{gathered}\text { 2-line } \\ \text { extended }\end{gathered}$ Irregular Freehand

\begin{tabular}{|c|c|c|c|c|c|c|c|}
\hline \multirow{2}{*}{\multicolumn{8}{|c|}{$\begin{array}{l}\text { Mean rank as best example for each Transformatio } \\
\text { Collapsed over the three shape categories }\end{array}$}} \\
\hline & & & & & & & \\
\hline $\begin{array}{c}\text { Himba } \\
\text { (Dani) }\end{array}$ & $\begin{array}{c}\mathbf{2 . 4 2} \\
(1.81)\end{array}$ & $\begin{array}{c}\mathbf{4 . 0 0} \\
(3.82)\end{array}$ & $\begin{array}{c}\mathbf{4 . 5 1} \\
(4.37)\end{array}$ & $\begin{array}{c}\mathbf{3 . 8 5} \\
(3.77)\end{array}$ & $\begin{array}{c}\mathbf{3 . 7 0} \\
(3.32)\end{array}$ & $\begin{array}{c}\mathbf{4 . 5 8} \\
(4.99)\end{array}$ & $\begin{array}{c}\mathbf{4 . 9 4} \\
(5.94)\end{array}$ \\
\hline \multicolumn{8}{|c|}{ By shape category } \\
\hline \multicolumn{8}{|l|}{ Circle } \\
\hline $\begin{array}{r}\text { Himba } \\
\text { Dani }\end{array}$ & $\begin{array}{c}\mathbf{2 . 1 8} \\
(1.18)\end{array}$ & $\begin{array}{c}\mathbf{3 . 7 3} \\
(4.00)\end{array}$ & $\begin{array}{c}\mathbf{5 . 0 9} \\
(3.63)\end{array}$ & $\begin{array}{c}\mathbf{5 . 0 0} \\
(4.09)\end{array}$ & $\begin{array}{c}\mathbf{3 . 3 6} \\
(3.22)\end{array}$ & $\begin{array}{c}4.27 \\
(5.54)\end{array}$ & $\begin{array}{l}\mathbf{5 . 3 6} \\
(6.72)\end{array}$ \\
\hline \multicolumn{8}{|l|}{ Square } \\
\hline $\begin{array}{r}\text { Himba } \\
\text { Dani }\end{array}$ & $\begin{array}{r}2.73 \\
(1.54) \\
\end{array}$ & $\begin{array}{c}4.91 \\
(3.91)\end{array}$ & $\begin{array}{c}3.91 \\
(4.28) \\
\end{array}$ & $\begin{array}{c}3.82 \\
(4.11) \\
\end{array}$ & $\begin{array}{c}3.45 \\
(3.56) \\
\end{array}$ & $\begin{array}{c}\mathbf{4 . 5 5} \\
(6.27)\end{array}$ & $\begin{array}{c}\mathbf{4 . 6 4} \\
(4.90)\end{array}$ \\
\hline \multicolumn{8}{|l|}{ Triangle } \\
\hline $\begin{array}{r}\text { Himba } \\
\text { Dani }\end{array}$ & $\begin{array}{c}2.36 \\
(2.69)\end{array}$ & $\begin{array}{c}\mathbf{3 . 3 6} \\
(3.54) \\
\end{array}$ & $\begin{array}{c}\mathbf{4 . 5 5} \\
(5.17) \\
\end{array}$ & $\begin{array}{r}\mathbf{3 . 7 3} \\
(3.07) \\
\end{array}$ & $\begin{array}{c}4.27 \\
(3.18)\end{array}$ & $\begin{array}{c}4.91 \\
(3.16)\end{array}$ & $\begin{array}{r}4.82 \\
(6.19) \\
\end{array}$ \\
\hline
\end{tabular}

Figure 1A. The 21 shapes used in Set 1 ( 7 each for square, circle, triangle). (a) the 'good' form (prototype for set 1) (b) gap; (c) line-to-curve; (d) single line extended; (e) two-lines extended; (f) irregular figure; (g) freehand.
(a)
(b)
(c)
(d)
(e)
(f)
(g)

squares
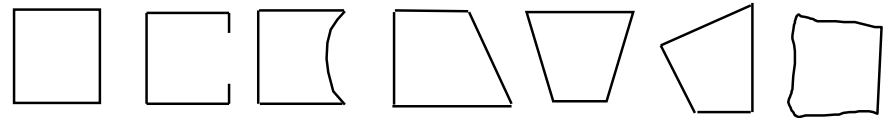

triangles
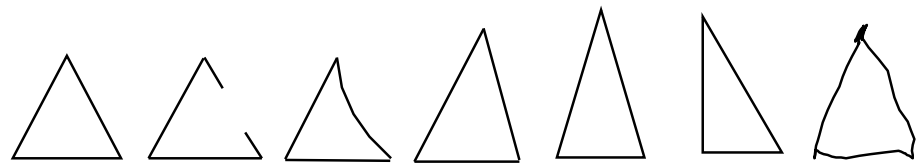

circles
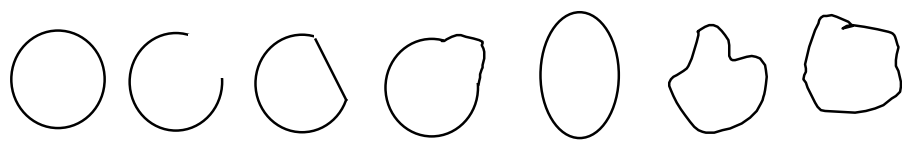
Figure 1B. The 21 shapes used in Set 2 (a) the 'good' form (gap closed); (b) gap (prototype for Set 2); (c) line-to-curve; (d) single line extended; (e) two-lines extended; (f) irregular figure; (g) freehand.
(a)
(b)
(c)

squares

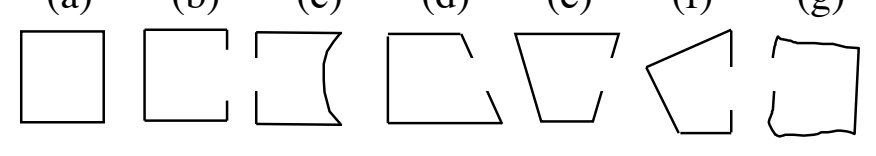

triangles
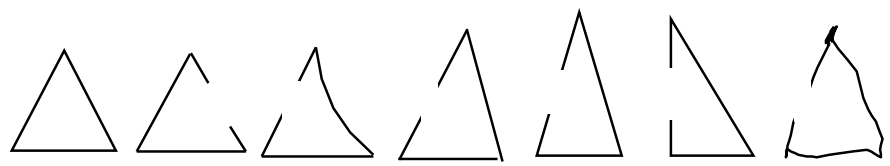

circles
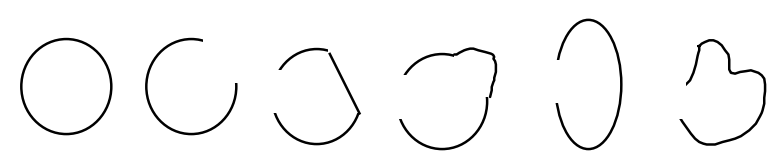

1 